\title{
Fundamentos conceituais para abordagens de gestão da inovação em bibliotecas
}

\author{
Murilo Mauro Silveira \\ William Barbosa Vianna \\ Ana Clara Cândido \\ Universidade Federal de Santa Catarina - UFSC, Brasil
}

REVIEW

\section{Resumo}

O objetivo do estudo é evidenciar os principais conceitos e abordagens sobre inovação para identificar as características e enquadramentos teóricos pertinentes aos estudos sobre inovação em Bibliotecas como organizações predominantemente públicas e sem fins lucrativos. Justifica-se pelo reconhecimento da importância da inovação nas Bibliotecas e pela necessidade de fundamentação teórica para o desenvolvimento do tema no âmbito da gestão da inovação cuja concentração se dá predominantemente na indústria de manufatura e relacionada a produtos. Trata-se de um ensaio teórico, de natureza qualitativa, conceitual e analítica. Espera-se com os resultados deste estudo possam contribuir na fundamentação teórica para o desenvolvimento de estudos futuros sobre a inovação em Bibliotecas.

Palavras-chave

Biblioteconomia; Epistemologia em Ciência da Informação; Gestão da Informação; Gestão da Inovação; Inovação em Bibliotecas

\section{Conceptual Foundations for Innovation Management approaches in Libraries}

\begin{abstract}
The objective of this study is to highlight the key concepts and approaches to innovation. Identifying characteristics and relevant theoretical frameworks to future studies on innovation in libraries as organization predominantly public and nonprofit. The main justification by the recognition of the importance of innovation in libraries and need to theoretical basis for the issue of development in the innovation management occurs predominantly in the manufacturing industry and related products. This is a theoretical essay, qualitative, conceptual and analytical nature. It is hoped the results of this study can contribute to the theoretical basis for the development of future studies on innovation in libraries.
\end{abstract}

Keywords

Epistemology in Information Science; Information Management; Innovation in Libraries; Innovation Management; Librarianship

\section{Introdução}

O atual ambiente econômico globalizado enseja, além da capacidade de adaptação e flexibilidade das organizações, o incremento de diferenciais que agreguem valor em produtos e ou serviços ofertados na percepção dos usuários.

Estes desafios apontam as discussões para necessidade de inovar e de como promover ou potencializar a inovação. De acordo com Stefanovitz (2011, p.1), "esta questão exige um conhecimento profundo de como ocorre o processo de inovação. E é neste ponto que se iniciam as dificuldades conceituais e práticas".

Com bibliotecas e unidades de informação sem fins lucrativos não é muito diferente. De acordo com Kostagiolas, Margiola e Avramidou (2011), estas organizações devem enfrentar as pressões econômicas e sociais, de forma que, neste contexto socioeconômico, seus serviços sejam geridos de forma a satisfazer as necessidades 
informacionais e atendimento das expectativas de seus usuários.

As possibilidades de inovação como forma de agregar valor aos serviços, para atender as necessidades e expectativas dos seus usuários é identificado por Georgy (2010a), quando afirma que as bibliotecas também podem posicionar o sucesso no futuro, devendo aderir a uma gestão moderna com métodos de gerenciamento baseados e adaptados para suas especificidades, como é a gestão da inovação. Por meio de seus estudos em bibliotecas, Georgy (2010b) observou que é necessário ter um olhar para o tema de gestão da inovação em Bibliotecas uma vez que o tema ganhou considerável importância nestes últimos anos.

Neste sentido, Damanpour, Walker e Avellanela (2009) afirmam que recentemente investigadores de inovação têm enfatizado as diferenças na natureza das atividades das organizações de manufatura e serviços, e a importância de desenvolvimento de modelos de inovação próprios para organizações de serviços. Em seu estudo, os autores forneceram evidências empíricas para este ponto de vista e demonstraram que as inovações de processo administrativo influenciam o desempenho organizacional nas instituições de serviços públicos.

Para Walker (2006), instituições públicas e governamentais procuram desenvolver a capacidade de inovar utilizando a inovação para alcançar níveis mais elevados, em termos de tipos e desempenho, tornando-se um ambiente fértil para o estudo da influência da inovação no desempenho do setor.

Nas bibliotecas, de acordo com Leonard e Clementson (2012), embora pesquisas publicadas sobre inovação sejam escassas, quando comparadas com pesquisas publicadas a partir de outras disciplinas, tais como negócios e economia, há uma riqueza de possibilidades para alavancar as invetigaçoes sobre o tema.

Há uma convergência na literatura de que várias barreiras impedem a inovação em bibliotecas, como por exemplo: a) estrutura hierárquica tradicional; b) a natureza de instituições sem fins lucrativos, isto é, entregam a comunidades e usuários apenas o valor social; c) o fato de serem organizações maduras ou envelhecidas, isto é, dificuldade em correr risco, experimentar e criar inovações por serem organismos maduros, d) dificuldades em replicar o sucesso de forma consistente e baseada em experiências do passado, (DEISS, 2004; LEONARD; CLEMENTSON, 2012; ROWLEY, 2011).

Fica evidente a necessidade de mais estudos orientados para identificar o que é representativo na gestão da inovação em bibliotecas e o potencial que possuem para contribuir para a temática da gestão da inovação em bibliotecas. Sobretudo, pois as pesquisas sobre inovação têm seguido um imperativo tecnológico e seus indicadores baseiam-se predominantemente em termos de novos produtos e outras saídas.

Situação confirmada por Damanpour, Walker e Avellanela (2009) ao afirmar que as empresas organizam principalmente seus esforços de atividades de inovação por meio de Pesquisa e Desenvolvimento (P\&D), e tem, portanto, focado em uma definição restrita a inovações de produtos, associado com a função de P\&D nas organizações de manufatura. Para os autores, inovações em empresas de serviços são escassas e sugerem uma adoção combinativa de diferentes tipos de inovações em diferentes partes da organização. Assim, para que aumente a sua capacidade para uma mudança adaptativa, uma vez que, as organizações devem se adaptar as pressões competitivas e ou institucionais promovidas pelo ambiente externo.

Nesse sentido, pergunta-se: de que forma caracterizar e fundamentar epistemologicamente os estudos sobre Inovação em Bibliotecas no campo da Ciência da Informação? O objetivo do estudo é evidenciar os principais conceitos e abordagens gerais sobre inovação para identificar as características e enquadramentos teóricos pertinentes aos estudos sobre inovação aplicados a Bibliotecas como organizações predominantemente públicas e sem fins lucrativos.

Justifica-se pelo reconhecimento da importância da inovação nas Bibliotecas e pela necessidade de fundamentação teórica para o desenvolvimento do tema no âmbito da gestão da inovação aplicada devido ao fato de que a concentração da produção intelectual tem se dado predominantemente na indústria de manufatura 
e relacionada a produtos.

Este estudo é caracterizado como um ensaio teórico, de natureza conceitual e analítica. E os resultados esperados são a fundamentação teórica para o desenvolvimento de estudos futuros sobre a inovação em Bibliotecas.

Quanto ao enquadramento metodológico, a abordagem é caracterizada como qualitativa, pois, de acordo com Creswell (2014), a visão de mundo do pesquisador é que dá sentido às interpretações em que o mundo é representado. No âmbito desta pesquisa, a análise e interpretação das variáveis de medição utilizadas na gestão da inovação se fazem presentes em dois contextos (geral e específico).

\section{Conceito e definição dos tipos de inovação}

É consensual a importância dos primeiros estudos de inovação para o desenvolvimento deste conceito e, neste âmbito o papel do economista Joseph Alois Schumpeter (1942), marcado como ponto de partida para a inserção do referido conceito. Desde então reconhecendo o papel da inovação como propulsora do desenvolvimento econômico das nações. No atual contexto, o termo "inovação" tem sido marcado nas mais diversas abordagens, prevalecendo as análises empíricas no âmbito empresarial. Esta ideia será aqui reforçada no intuito de analisar o desenvolvimento e gestão de inovações no contexto das bibliotecas.

De acordo com Tidd (2001), estudos de inovação têm sido baseados e adotados por uma ampla gama de disciplinas, como Economia, Geografia, Sociologia, Psicologia, acarretando em diferentes métodos, definições e exemplos. Pesquisas nestas áreas, de acordo com suas diferentes visões, contribuíram para o entendimento da inovação em seu nível macro (sociedade, sistema econômico, indústria) e seu nível micro (empresa). Portanto, a gestão da inovação compreende o nível micro, onde estão envolvidas as organizações de todo tipo.

Devido aos equívocos nos conceitos de inovação e invenção, esta é uma das primeiras definições que emergem nas conversas neste âmbito. Sobre esta distinção, reforça-se que uma invenção só passará a ser uma inovação se o mercado reconhecer o valor. Portanto, nem todas as invenções poderão ser consideradas inovações (Manual de Oslo, 2005).

O conceito de inovação é bastante amplo, para os autores Harrington e Voehl (2013) a palavra inovação, de origem latina (Novus) significa novo, (Novare) refere-se a renovar, criar, inventar. Em seus estudos, estes autores, assim como esta pesquisa, se basearam na definição do conceito de inovação segundo o Manual de Oslo (2005). A definição apresentada por este é: inovação é a implementação de um produto novo ou significadamente melhorado, ou um novo processo ou um novo método de marketing ou um novo método organizacional.

Harrington e Voehl (2013) distinguem que a inovação é o processo de criar algo novo, que tenha significativo valor a um indivíduo, organização, indústria ou sociedade, de forma que a palavra inovação é uma criação que tem valor significativo e amplo. É preciso que tenham potencial para operacionalização e criação de valor, tornando-se assim, inovações. Esse potencial desenvolvido pode trazer bons resultados para indivíduos, organizações, sociedade e governos.

Outra terminologia importante para abordagens sobre inovação é o grau de novidade, podendo ser radical ou incremental. A inovação radical tem custos mais elevados e maiores riscos, pois normalmente demanda mais investimento em P\&D. No entanto, se houver êxito no seu desenvolvimento o retorno é muito superior ao desenvolvimento de inovações incrementais. Por sua vez, as inovações incrementais correspondem a alterações e melhorias no produto e/ou serviço. Tendo em vista que partem de algo já pronto são mais fáceis de serem desenvolvidas em comparação às inovações radicas. 
Para Nilsson e Ritzén (2014), as oportunidades para inovação radical são estimuladas por estruturas informais, flexíveis, que surgem a partir do emprego e engajamento em iniciativas de apoio substancial em cima de uma gestão focada no mercado. A inovação incremental trata do resultado, controle e planejamento tradicional de exercícios, funções.

A inovação, como observado possui conceitos e entendimentos heterogêneos. Os tipos de inovação também possuem estas distinções, por grupos ou categorias. Uma destas categorizações foi proposta por GarcíaZamora, González-Benito e Muñoz-Gallego (2013) quando classificaram em: grau de novidade; cultura e operações e; unidimensional e multidimensional. Segundo estes autores, a primeira classificação de tipos de inovação refere-se ao grau de novidade envolvido. Divide-se em inovação radical e inovação incremental.

A inovação radical implica na criação de novos produtos e/ou processos para um novo mercado. Já a inovação incremental melhora um produto e/ou processo já existente. A segunda classificação refere-se à inovação cultural e operacional. Criam-se novas iniciativas, ideias e decisões e torna-se operacional quando aplicada. A terceira categoriza por tipo de inovação de acordo com sua abordagem, que pode ser unidimensional (produto ou processo) ou multidimensional (marketing organizacional, processos e gestão).

Em se tratando da primeira categorização, isto é, o grau de novidade envolvido na inovação, Harlow (2011); Jenkins (2014); Nilsson e Ritzén (2014); Tidd (2001); consideram que o conceito de inovação incremental e radical pode ser considerado como pontos ao longo de um continuum, que se diferencia como adaptações de ideias existentes em matéria de inovações incrementais e adoção de inovações de conceitos novos e distintos como inovações radicais.

A segunda categorização refere-se à cultura e operações. Parte-se do princípio que as organizações devem promover uma cultura voltada para a inovação, cuja operação se beneficiará com as ideias e iniciativas provindas por um ambiente favorável a inovação.

A terceira categoria trata da concepção de inovações de acordo com sua tipologia. Dentre os estudos que consideram como sendo Unidimensional ou Multidimensional, destacam-se Bisbe e Malagueno (2009); Cormican e Sullivan (2004); Giannopoulos (2015); Harrington e Voehl (2013); Hult, Hurley e Knight (2004); Tidd (2001). Para melhor representar esta distinção, o Quadro 1 demonstra a característica de cada uma das dimensões.

\begin{tabular}{|l|l|l|l|}
\hline \multicolumn{1}{|c|}{ Dimensão } & \multicolumn{1}{|c|}{ Conceito } \\
\hline \multirow{3}{*}{ Unidimensional } & $\begin{array}{l}\text { A inovação de produto é aqui entendida a partir de uma perspectiva de saída, e é } \\
\text { definida com o desenvolvimento e lançamento de produtos que estão em algum aspecto } \\
\text { único, objetivo ou distintivo dos produtos existentes. }\end{array}$ & $\begin{array}{l}\text { Malagueno } \\
\text { (2009) }\end{array}$ \\
\hline & $\begin{array}{l}\text { A inovação de produtos por si só não pode ser suficiente para assegurar o crescimento } \\
\text { futuro. Qualquer estratégia de negócios deve se concentrar em outros setores de } \\
\text { inovãa, bem, como serviços, a fim de estabelecer uma posição competitiva dentro da } \\
\text { indústria. }\end{array}$ & $\begin{array}{l}\text { Giannopoulos } \\
\text { (2015) }\end{array}$ \\
\hline & $\begin{array}{l}\text { A inovação de produto é um processo contínuo e integrado a um crescente número de } \\
\text { diferentes competências dentro e fora das fronteiras organizacionais. Simplificando, é o } \\
\text { processo de transformar oportunidades de negócios em produtos tangíveis e ou } \\
\text { serviços. }\end{array}$ & $\begin{array}{l}\text { Sullivan } \\
\text { (2004) }\end{array}$ \\
\hline
\end{tabular}

Quadro 1- Inovação por dimensões (Continua) 


\begin{tabular}{|c|c|c|}
\hline Dimensão & Conceito & Referências \\
\hline \multirow{3}{*}{ Unidimensional } & $\begin{array}{l}\text { Inovação de produto refere-se à concepção, desenvolvimento e oferta de bens e } \\
\text { serviços. Segundo a Organização para a Cooperação Econômica e Desenvolvimento } \\
\text { Econômico (OCDE): uma inovação de produto é a introdução de um bem ou serviço } \\
\text { novo ou significativamente melhorou no que diz respeito às suas características ou usos } \\
\text { pretendidos. }\end{array}$ & $\begin{array}{l}\text { Harrington; } \\
\text { Voehl (2013) }\end{array}$ \\
\hline & $\begin{array}{l}\text { Inovação de processo trata-se de métodos para aumentar produtividade e corte de } \\
\text { custos. É definida pela OCDE como a implementação de um método de produção ou } \\
\text { distribuição novo ou significativamente melhorado. Isso inclui mudanças significativas } \\
\text { em técnicas, equipamentos e / ou software. }\end{array}$ & $\begin{array}{l}\text { Harrington; } \\
\text { Voehl (2013) }\end{array}$ \\
\hline & $\begin{array}{l}\text { A inovação pode assumir duas formas básicas: a inovação de produtos, que é } \\
\text { alterações nos produtos ou serviços que uma organização oferece; e a inovação de } \\
\text { processo que é alterações nos produtos e formas de serviço que são criados e } \\
\text { entregues. }\end{array}$ & Tidd (2001) \\
\hline \multirow{3}{*}{ Multidimensional } & $\begin{array}{l}\text { Uma inovação pode ser um novo produto ou serviço, uma nova produção, processo, ou } \\
\text { uma nova estrutura ou sistema administrativo. }\end{array}$ & $\begin{array}{l}\text { Hult; Hurley; } \\
\text { Knight (2004) }\end{array}$ \\
\hline & $\begin{array}{l}\text { Ao contrário da maioria das empresas, que se voltam para produto ou design de } \\
\text { serviços para melhorar a satisfação do cliente, os líderes perseguem quatro tipos de } \\
\text { inovação. De acordo com especialistas em inovação, existem basicamente quatro tipos } \\
\text { de estratégias de inovação que as organizações precisam se preocupar: produto, } \\
\text { processo, marketing e inovações gerenciais. }\end{array}$ & $\begin{array}{l}\text { Harrington; } \\
\text { Voehl (2013) }\end{array}$ \\
\hline & $\begin{array}{l}\text { Gestão da inovação, gere a inovação usando vários métodos e programas, melhora a } \\
\text { forma como a organização é administrada. A gestão da inovação pode ajudar a } \\
\text { alcançar ambas as diferenciações com baixo custo, vantagem competitiva através da } \\
\text { melhoria da eficiência e eficácia dos esforços logísticos para atingir metas corporativas. }\end{array}$ & $\begin{array}{l}\text { Harrington; } \\
\text { Voehl (2013) }\end{array}$ \\
\hline
\end{tabular}

Quadro 1- Inovação por dimensões (Final)

A partir do Quadro 1, é possível identificar e categorizar os fundamentos conceituais para o desenvolvimento da inovação em bibliotecas a partir uma ou mais dimensões, sendo que em vista o contexto estudado no âmbito deste estudo, as bibliotecas, é útil também situar o desenvolvimento dos estudos considerando o marco teórico proposto pela definição do Manual de Oslo (2005) que é a principal referência para padronização dos conceitos relativos à inovação em nível internacional. Com relação aos tipos de inovação, o Manual de Oslo (2005) apresenta as seguintes definições:

- Inovação de produto - introdução de um bem ou serviço novo ou significadamente melhorado;

- Inovação de processo - implementação de um método de produção ou distribuição novo ou significadamente melhorado;

- Inovação de marketing - implementação de um método de marketing com mudanças significativas na concepção de produto ou sua comercialização e;

- Inovação organizacional - implementação de um novo método organizacional na prática dos negócios da empresa, na organização do seu local de trabalho ou em suas relações externas. 
A clareza e distinção conceitual dos tipos de inovação é fundamental para que as organizações possam utilizar as diferentes concepções em seu beneficio e no caso das bibliotecas mais ainda, uma vez que no âmbito de outras disciplinas poderiam ocorrer dificuldades em considerar a utilidade ou ocorrência da inovação em organizações sem fins lucrativos, como se a mesma se limitasse à inserção de novos produtos no mercado econômico. Também é relevante reconhecer a evolução e o gerenciamento da inovação no contexto organizacional, uma vez que propicia aos gestores de organizações um entendimento acerca da necessidade de inovar em sua empresa e de melhor se orientar para inovar.

Harrington e Voehl (2013) afirmam que a uma inovação de sucesso significa muito mais que apenas criar algo novo, propor ideias. Significa ser capaz de se mover em novas soluções e defendê-las em resultados que proporcionem valor ao cliente, melhorando processos, e construindo novas oportunidades.

Para Adams, Bessant e Phelps (2006), as organizações precisam ser capazes para fornecer liberdade suficiente para permitir a exploração de possibilidades criativas, mas faz se necessário o controle suficiente para gerenciar a inovação de forma eficaz e eficiente. Neste contexto, a inovação deve ser explorada de forma que a organização possa usufruir de sua total vantagem, contudo, é necessária uma gestão atenta para seus benefícios e controle de riscos e incertezas.

Na opinião dos autores García-Zamora, González-Benito e Muñoz-Gallego (2013), a implementação de iniciativas inovadoras está ligada à capacidade das organizações responderem a mudanças e as oportunidades ambientais. Esta mesma visão é compartilhada por Hult, Hurley e Knight (2004), quando afirmam que a inovação é um meio para uma organização se modificar, mesmo com uma resposta as alterações que ocorrem no seu ambiente interno e externo.

E Tidd (2001), por sua vez, descreve que empresas inovadoras são mais protegidas contra quedas cíclicas. Estas afirmações são fundamentais para o desenvolvimento da temática da inovação em bibliotecas, pois apontam para um sentido pouco explorado do tema.

Para Rowley (2011), a gestão da inovação tem um âmbito mais vasto do que a gestão da mudança e aplica-se a alterações em todo o produto, serviço, processo e organizações, bem como, considera o ambiente organizacional. Portanto, o ambiente organizacional é visto como fator relevante para a gestão da inovação.

\section{Elementos para a Gestão da Inovação Organizacional}

De acordo com Hagedoorn e Cloudt (2003), pesquisas nos últimos anos retratam que a inovação tem aumentado e tornou-se uma necessidade para as organizações. Giannopoulos (2015); Gómez, López e González (2012); Gumilar, Zarnić e Selih (2011) concordam que a novidade se tornou uma necessidade, e é isto que define as bases para o crescimento de uma organização e no atual ambiente econômico induzem as organizações a considerar a inovação como recurso estratégico, que permite criar vantagens sustentáveis.

Entretanto, para Damanpour, Walker e Avellanela (2009) o sucesso nas organizações de serviços não segue uma trajetória tecnológica, mas dependem da adoção de dois tipos de inovação: tecnológica e não tecnológica. As pesquisas que contaram com os modelos de co-evolução do produto à base de tecnologias e inovações de processo para explicar as consequências da inovação e sua adoção em organizações, tem demonstrado que a co-adopção de diferentes tipos de inovação pode explicar melhor as consequências do desempenho da inovação nas organizações de serviço. O referido estudo forneceu evidência empírica para este ponto de vista e demonstrou que o co-adoção de serviço, processo tecnológico e inovações de processo administrativo influenciam o desempenho organizacional nas organizações de serviços públicos.

Damanpour, Walker e Avellanela (2009) afirmam ainda que dado o foco em atender as necessidades dos clientes no setor de serviços, a natureza da inovação em serviços é melhor compreendida através da relação 
com o usuário do serviço. Para os autores, a definição de inovação em serviços é compreendida como a introdução de novos serviços para os clientes existentes ou novos.

Para entender a inovação dentro das organizações, e reconhecer a evolução da inovação, o estudo de Jerry e Donatella (2013) apresenta um breve relato sobre sua historicidade. Os autores relatam que desde a década de 1980, houve um movimento no sentido de uma abordagem sistemática para introduzir a inovação organizacional. Esta abordagem tem como objetivo gerenciar os locais de trabalho. Portanto, o resultado da inovação reflete um amplo foco estratégico das organizações.

Ainda segundo os autores, até a década de 1990, as inovações incluíram a fabricação de robôs, automatização de processos, máquinas, computadores, isto é, ligadas às tecnologias, consideradas como inovações radicais. A partir de 1990 preocupou-se com inovações que ocorriam em produtos já montados, por exemplo, carros, onde se introduziu inovações do tipo: airbags, sistemas de posicionamento, eficiência em relação ao consumo.

Em outro contexto, Harrington e Voehl (2013) fazem uma comparação entre inovação e qualidade ao confrontar a evolução destas áreas. Para os autores, hoje em dia a prática de inovação é semelhante à forma com que as empresas se aproximaram da qualidade no início de 1980. Naquela época, a qualidade era tratada em um departamento da empresa, mas que agora se tornou uma responsabilidade de toda a instituição. De acordo com os autores, hoje em muitas organizações, a inovação ainda é tratada em departamentos, principalmente em P\&D ou marketing. Mas que, assim como a qualidade, espera-se que a inovação seja responsabilidade de todos Para Adams, Bessant e Phelps (2006), as organizações precisam ser capazes para fornecer liberdade suficiente para permitir a exploração de possibilidades criativas, mas faz se necessário o controle suficiente para gerenciar a inovação de forma eficaz e eficiente. Neste contexto, a inovação deve ser explorada de forma que a organização possa usufruir de vantagens, contudo, é necessária uma gestão atenta para seus benefícios e controle de riscos e incertezas.

Tidd (2001) afirma que várias décadas de pesquisas em gestão da inovação não conseguiram fornecer achados consistentes ou conselhos coerentes para os gerentes. O autor defende em seu estudo que a gestão da inovação (melhores práticas) depende de uma série de fatores que precisam de melhores caracterizações das contingências tecnológicas e de mercado, que de certa forma afetam as oportunidades e restrições para a inovação. Esses fatores podem ser mais bem direcionados a partir de modelos de gestão da inovação.

Podem-se observar no fragmento da literatura, a seguir, vários aspectos relevantes para gestão da inovação organizacional.

Descrição

A gestão da inovação em organizações melhora a rentabilidade em curto prazo quando se trata de uma contínua inovação dentro da organização. Gera melhor rentabilidade, pois toda a organização que se envolve em um ambiente de mudança e melhoria constante precisa alcançar uma maior eficiência.

Existe uma necessidade contínua de explorar as oportunidades e construir um saudável e próspero futuro, criar novos fluxos de receita e riqueza, descobrir novas soluções, e transformar a nossas organizações, indústrias e sociedades. Esta necessidade leva-nos a focar em gestão da inovação.

\section{Referências}

García-Zamora; González-Benito; MuñozGallego (2013)

Harrington; Voehl (2013)

Quadro 2 - Gestão da inovação e sucesso organizacional (Continua) 
A adoção da gestão da inovação é um meio para a mudança organizacional. Oportunidades, ameaças e mudanças no ambiente, motivam as organizações a procurarem mudanças adaptativas. Assim, através da adoção de inovações ao longo do tempo as organizações pretendem ajustar as suas funções internas e externas, para que pudessem responder aos ambientes, demandas, para operar de forma eficiente e eficaz, e manter ou melhorar seu desempenho.

Gestão da inovação pode funcionar como um acelerador de mudança, através do desenvolvimento novas formas de gerir a mudança e estresse, bem como melhores sistemas de Harrington; Voehl (2013) informação.

O foco em inovação tem sido sempre um fator crítico para o sucesso de organizações. Hoje, no entanto, isso não requer somente o desenvolvimento e implementação de novas tecnologias, mas também, uma compreensão de relações complexas serem geridas de forma a desenvolver novos produtos, serviços.

As empresas precisam adotar estratégias de gestão da inovação, para que o rendimento criação, desenvolvimento, teste e implementação de uma invenção possa se transformar em uma inovação.

Damanpour; Walker;

Avellaneda (2009)

\section{Quadro 2 - Gestão da inovação e sucesso organizacional (Final)}

Como se pode verificar pelo quadro 2, diversas possibilidades teóricas e práticas baseadas na literatura podem ser aportadas para estudos sobre inovação em biblioteca de forma fundamentada e epistemologicamente coerente.

Em se tratando de desenvolvimento modelos de gestão da inovação nas bibliotecas, considera-se como condição necessária considerar que muitos estudiosos têm procurado identificar atividades chaves de processos da gestão da inovação, alguns dos quais são pré-estabelecidos como modelos lineares, outros possuem caráter dinâmico e recursivos por loops de feedback, sendo que estes modelos são limitados a partir de uma perspectiva de medição (ADAMS, BESSANT E PHELPS, 2006).

Ainda para os autores (2006), outros modelos têm sido principalmente gerados em contextos de tecnologias, portanto, também não são generalizados; por sua vez, outros não consideram a difusão da inovação organizacional e sua conexão sócio técnica com todos os aspectos organizacionais ou níveis de integração pretendidos.

\section{Gestão da inovação em bibliotecas}

De acordo com Jianzhong e Chen (2013), uma boa biblioteca, nos velhos tempos dourados, era um espaço físico com dezenas de milhares de itens de livros, onde havia espaço de leitura suficiente para as pessoas a encontrar e ler livros confortavelmente, e que era capaz de atender a demanda dos usuários. Mas hoje, a biblioteca tem de ser agressiva e dinâmica. Esta dinamicidade deve-se a diversos fatores.

Segundo estes autores, três fatores impactaram as bibliotecas nos últimos 30 anos. O primeiro em meados de 1980, quando o crescimento da internet foi acelerado pela combinação: computador, informação, comunicação e tecnologia. O segundo veio com a virada do século XXI, por meio da internet e o aumento explosivo das informações eletrônicas. O terceiro está relacionado com a ideia de uma sociedade sem papel, onde pessoas estão se questionando sobre usar o meio digital ou o tradicional papel. 
Ainda conforme Jianzhong e Chen (2013), hoje a biblioteca está no período de transição para uma idade eletrônico-e-globalizada. As bibliotecas são supostas guardiões da informação e do conhecimento, em vez de encarregados de pilhas de livros. A crescente demanda por uma mudança de papel em processamento de conhecimento, conteúdo, mineração e interação do usuário provocou motivações para bibliotecas reformularem suas estratégias de gestão e de serviços.

Em seu estudo, Rowley (2011) declara que houve pouca discussão sobre inovação e seus processos por parte dos profissionais da informação, bem como na literatura acadêmica. Já Leonard e Clementson (2012) afirmam que existem relativamente poucos artigos de pesquisas em que o foco trata sobre a estrutura organizacional de bibliotecas acadêmicas que incentivem a inovação.

Jianzhong e Chen (2013) concluíram que as bibliotecas de hoje precisam de transformações fundamentais através das práticas de inovação, em vários aspectos e dimensões, para manter-se nos tempos atuais. Para tanto, faz-se necessário o entendimento do contexto em que as bibliotecas convivem para melhor compreensão desta nova dinâmica.

Para Fingerle e Fingerle (2012), está claro que a inovação deve estar nos objetivos das bibliotecas, e que o sucesso da inovação é que vai decidir sobre sua aceitação no mercado, neste caso, sua aceitação pelos clientes. Esta perspectiva também é corroborada por Harbo e Hansen (2012) quando afirmam que as bibliotecas querem melhorar a forma como elas servem as necessidades de seus usuários, devendo inovar seus serviços e instalações.

Já Georgy (2012) aponta que a gestão estratégica da inovação é muito importante em bibliotecas. Informa que dentro da Biblioteconomia e Ciência da Informação, bem como em instituições menores (pequenas e médias empresas, públicas ou privadas), as pessoas são atribuídas a incorporar a gestão estratégica para que o potencial destas instituições possa ser aumentado.

Para Rowley (2011) algumas das inovações são conduzidas pela oportunidade proporcionada pelas novas inovações tecnológicas de outras organizações, em conjunto com as mudanças nas expectativas e comportamento de consumo. O autor classifica as inovações de acordo com:

i) facilitadas pela tecnologia de informação, impulsionado por mudanças políticas e de mercado;

ii) não afetadas por plataformas tecnológicas, mas que representam por exemplo, envolvimento de inovações em comunidades tais como: serviços para grupos desfavorecidos, grupos de leitura e biblioterapia.

Em relação aos exemplos de inovações que consideram os aspectos tecnológicos, Munro et al. (2011) em seu estudo sobre uso de dispositivos móveis, acredita que as potenciais iniciativas e desenvolvimentos durante a pesquisa evidenciaram que o uso dos dispositivos móveis em serviços de bibliotecas tem recursos poderosos para transformar o acesso à informação em algo inovador e eficaz.

Para Kostagiolas, Margiola e Avramidou (2011), as bibliotecas experimentam de forma inovadora as ferramentas de tecnologia da informação para criar novos serviços para o seu público, isto é, projetos e hospedagem em web site, aulas gratuitas sobre diversos temas de como utilizar as tecnologias (pesquisas on-line, preparar curriculum vitae, procurar empregos através de redes de informação).

As inovações que não consideram os aspectos tecnológicos podem ser observadas por Kostagiolas, Margiola e Avramidou (2011), quando afirmam que muitas bibliotecas organizam eventos para promover leitura, cultura, criatividade e inovação. Os autores acreditam que os serviços inovadores incluem atividades relacionadas com a 
aprendizagem, educação, formação, desenvolvimento econômico, social, e-governo, identidade cultural, coesão e inclusão social, de forma a contribuir para o desenvolvimento de competências humanas, ampliando as oportunidades econômica e social de que precisam.

Rowley (2011), em seu estudo, propôs um modelo ligado a estratégias de inovações em bibliotecas. Este modelo considera sete aspectos da inovação que poderiam ser úteis para a estratégia de inovações: capacidade e cultura de inovação; carteira de inovações; inovações de processo; liderança para inovação; equipes criativas e inovadoras; inovação e colaboração aberta e; envolvimento do usuário em inovação. Assim, o autor acredita que este modelo de sete facetas atende a uma abordagem mais holística e dinâmica para atividades de inovações dentro de bibliotecas.

Para Leonard e Clementson (2012), uma conclusão fundamental é que o suporte organizacional é necessário para que o bibliotecário prospere na produção de inovações. Afirmam que os bibliotecários querem que suas ideias sejam ouvidas, e que para isso, é essencial a atenção e apoio por parte da administração das bibliotecas. Desta forma, acredita-se que as bibliotecas devem prover subsídios para que os bibliotecários sejam profissionais inovadores, mas que, são administradas por gestores (bibliotecários ou não bibliotecários), e, portanto, estes também são responsáveis por promover a gestão da inovação organizacional.

Os autores prosseguem que o empreendedorismo que gera a inovação, e que é necessário para sobreviver e competir no mercado competitivo são inibidos pela estrutura hierárquica tradicional presente nas bibliotecas. Os mesmos sugerem que, apesar de os bibliotecários possuírem um espírito empreendedor, as estruturas organizacionais presentes em bibliotecas podem criar barreiras à inovação, sendo esse também um desafio terórico e prático a ser explorado pela ciência da informação.

Outro desafio é apresentado por Rowley (2011), para quem não surpreende que as bibliotecas, a maioria das quais são organizações do setor público, têm que trabalhar duro e serem persistentes para alcançar inovações, e mais ainda, incorporar a inovação na prestação de serviços.

E por fim, Deiss (2004), afirma que o desenvolvimento de métodos para reduzir estas barreiras, bem como, aumentar a confiança pessoal, compromisso e habilidades são importantes para que as bibliotecas se tornem verdadeiramente inovadoras.

\section{Considerações finais}

Tendo em consideração os tipos de inovação apresentados ao longo deste estudo, observa-se que a inovação do tipo organizacional se aproxima de forma mais direta com as iniciativas presentes no contexto das bibliotecas. Neste tipo de inovação, as iniciativas e boas práticas e adotadas permitem que a organização melhore seu desempenho frente aos desafios enfrentados e apresente insights importantes para o fortalecimento de uma cultura de inovação. Sendo o desafio destas instituições em lidarem com resistência à mudanças, normas e procedimentos pouco flexíveis e o interesse das pessoas e usuários sobre os serviços prestados.

Nestes termos, importante mencionar que o Manual de Oslo (2005) apresenta que, a inovação organizacional não serve apenas para apoiar as inovações de produtos e ou serviços, processos. Este tipo de inovação pode impactar o desempenho das empresas, melhorando sua qualidade e eficiência do trabalho, acentuar a troca de informações e refinar a capacidade empresarial de aprender e utilizar conhecimentos e tecnologias.

Considera-se, portanto, que a gestão da inovação em bibliotecas necessita de desenvolvimento especifico, de acordo com a natureza, finalidade e especificidade da organização, seja ela pública ou privada, especializada ou não e outras características, de forma que modelos e indicadores genéricos demonstram-se inadequados. Têmse ainda que o âmbito mais adequado para para o desenvolvimento de gestão da inovação em bibliotecas 
aponta para a inovação organizacional como dimensão mais profícua para fundamentação teórica devido à especificidade da organização.

\section{Referências}

ADAMS, R.; BESSANT, J.; PHELPS, R. Innovation management measurement: A review. International Journal of Management Reviews, v. 8, n. 1, p. 21-47, 2006.

BISBE, J.; MALAGUEÑO, R. The choice of interactive control systems under different innovation management modes. European Accounting Review, v. 18, n. 2, p. 371-405, 2009.

CORMICAN, K.; O'SULLIVAN, D. Auditing best practice for effective product innovation management. Technovation, v. 24, n. 10, p. 819-829, 2004.

CRESWELL, J. W. Research Design: Qualitative, Quantitative, and Mixed Methods Approaches. Sage Publications, CA. 2014.

DAMANPOUR, F.; WALKER, R. M.; AVELLANEDA, C. N. Combinative effects of innovation types and organizational performance: A longitudinal study of service organizations. Journal of Management Studies, v. 46, n. 4, p. 650-675, 2009.

DEISS, K. J. Innovation and Strategy: Risk and Choice in Shaping User-Centered Libraries. Library Trends, v. 53, p. 17-32, 2004.

FINGERLE, V. B.; FINGERLE, B. Innovation zum Mitmachen: Die Open Innovation-Kampagnen der ZBW Mit Open Innovation gemeinsam mit Kundinnen und Kunden Neuerungen in Bibliotheken fördern. Bibliothek, v. 36, n. 3, p.346-352, 2012.

GARCÍA-ZAMORA, E.; GONZÁLEZ-BENITO, O.; MUÑOZ-GALLEGO, P. A. Organizational and environmental factors as moderators of the relationship between multidimensional innovation and performance. Innovation, v. 15, n. 2, p. 224-244, 2013.

GEORGY, U. Erfolg durch Innovation. B.I.T. online Innovativ, Band 29. Wiesbaden, Dinges\&Frick. 2010a.

Qualität im Service- und Dienstleistungs management von Bibliotheken durch erfolgreiches Innovationsmanagement. B.I.T. online Innovativ, Band 30. Wiesbaden, Dinges \& Frick, p. 33-55, $2010 \mathrm{~b}$.

GIANNOPOULOS, A. Performance Management as a Process of Promoting Innovation in Software Industry. Procedia-Social and Behavioral Sciences, v. 175, p. 401-407, 2015.

GÓMEZ-C., J.; LÓPEZ-V., E.; GONZÁLEZ S., B. Management Control Systems, Innovative Capability Performance, and ISO Certification: Modelling their Effects on Organizational Performance. Revista Galega de Economía, v. 23. p. 245-270, 2012.

GUMILAR, V.; ZARNIĆ, R.; SELIH, J. Increasing Competitiveness of the Construction Sector by Adopting Innovative Clustering. Engineering Economics, v. 22, n. 1, p. 41-49, 2011.

HAGEDOORN, J.; CLOUDT, M. Measuring innovative performance: is there an advantage in using multiple indicators? Research policy, v. 32, n. 8, p. 1365-1379, 2003.

HARBO, K.; HANSEN, T. V. Getting to Know Library Users Needs - Experimental Ways to User-centred Library Innovation. Liber Quarterly, v. 21, n. 3/4, p. 367-385, 2012.

HARLOW, H. Developing a knowledge strategy using tacit knowledge measurement: implications for the balanced scorecard innovation and learning perspective. In: Proceeding of the 12th European Conference of Knowledge Management. University of Passau, Germany. UK: Academic Publishing. p. 416-423, 2011.

HARRINGTON, H. J.; VOEHL, F. Innovation Management: A Breakthrough Approach to Organizational Excellence-Part 1. International Journal of Innovation Science, v. 5, n. 4, p. 213-224, 2013.

HULT, G. T. M.; HURLEY, R. F.; KNIGHT, G. A. Innovativeness: Its antecedents and impact on business performance. Industrial marketing management, v. 33, n. 5, p. 429-438, 2004.

JENKINS, M. Innovate or Imitate? The Role of Collective Beliefs in Competences in Competing Firms. Long Range Planning, v. 47, n. 4, p. 173-185, 2014.

JERRY, C.; DONATELLA, C. A tale of two strategies: Framework for evaluating human resource management and innovation in Australia-Lessons for China. In: Management Science and Engineering (ICMSE), International Conference on. IEEE, 2013. p. $1295-1307,2013$.

JIANZHONG, W.; CHEN, X. Transition and transcendence: the innovative development of Shanghai Library. Library Management, v. 34, n. 1/2, p. 20-30, 2013. 
KOSTAGIOLAS, P.; MARGIOLA, A.; AVRAMIDOU, A. A library management response model against the economic crisis: The case of public libraries in Greece. Library Review, v. 60, n. 6, p. 486-500, 2011.

LEONARD, E.; CLEMENTSON, B. Business Librarians and Entrepreneurship: Innovation Trends and Characteristics. New Review of Information Networking, v. 17, n. 1, p. 1-21, 2012.

MANUAL DE OSLO. Proposta de diretrizes para coleta e interpretação de dados sobre inovação tecnológica. OECD, 1997. Traduzido pela FINEP - Financiadora de Estudos e Projetos em 2005.

MUNRO, K., STEVENSON, K., STENSON, R., WALKER, W., FISHER, C. Planning for the mobile library: a strategy for managing innovation and transformation at the University of Glasgow. Library Serials, v.24, p.26-31, 2011

NILSSON, S.; RITZÉN, S. Exploring the Use of Innovation Performance Measurement to Build Innovation Capability in a Medical Device Company. Creativity and Innovation Management, v. 23, n. 2, p. 183-198, 2014.

ROWLEY, J. Should your library have an innovation strategy? Library Management, v. 32, n. 4/5, p. 251-265, 2011.

SCHUMPETER, J.A. Capitalism, Socialism and Democracy. New York: Harper \& Row, 1942.

STEFANOVITZ, J.P. Contribuições ao estudo da gestão da inovação: proposição conceitual e estudo de casos. São Carlos, 2011. Tese (Doutorado) - Escola de Engenharia de São Carlos, Universidade de São Paulo, 2011.

TIDD, J. Innovation management in context: environment, organization and performance. International Journal of Management Reviews, v. 3, n. 3, p. 169-183, 2001.

WALKER, Richard M. Innovation type and diffusion: An empirical analysis of local government. Public administration, v. 84, n. 2, p. 311-335, 2006. 


\section{Dados dos autores}

Murilo Mauro Silveira

Bibliotecário. Mestre em Ciência da Informação. Universidade Federal de Santa Catarina - UFSC.

willscut2@hotmail.com

William Barbosa Vianna

Professor do Departamento de Ciência da Informação. Universidade Federal de Santa Catarina - UFSC.

wowilliam@hotmail.com

\section{Ana Clara Cândido}

Professora Adjunta do Departamento de Ciência da Informação. Universidade Federal de Santa Catarina UFSC.

acc.anaclara@gmail.com

Recibido - Received: 2016-09-29

Aceitado - Accepted: 2017-03-19

\section{(c) EY}

This work is licensed under a Creative Commons Attribution 4.0

United States License.

\section{ULIS D-Sorke}

This journal is published by the University Library System of the University of Pittsburgh as part of its

D-Scribe Digital Publishing Program and is cosponsored by the University of Pittsburgh Press. 